\title{
Synchronal Algorithm and Cyclic Algorithm for Hierarchical Fixed Point Problems and Variational Inequalities
}

\author{
Peichao Duan \\ College of Science, Civil Aviation University of China, Tianjin 300300, China \\ Correspondence should be addressed to Peichao Duan; pcduancauc@126.com
}

Received 26 May 2013; Accepted 23 August 2013

Academic Editor: Sehie Park

Copyright (c) 2013 Peichao Duan. This is an open access article distributed under the Creative Commons Attribution License, which permits unrestricted use, distribution, and reproduction in any medium, provided the original work is properly cited.

\begin{abstract}
We propose synchronal algorithm and cyclic algorithm based on the general iterative method for solving a hierarchical fixed point problem. Under suitable parameters, the iterative sequence converges strongly to a common fixed point of $N$ nonexpansive mappings and also the unique solution of a variational inequality. The results presented in this paper improve and extend the corresponding results reported recently by some authors. Furthermore, a numerical example is given to demonstrate the effectiveness of our iterative schemes.
\end{abstract}

\section{Introduction}

Let $H$ be a real Hilbert space with an inner product $\langle$,$\rangle and its$ induced norm $\|\cdot\|$. Let $C$ be a nonempty, closed, and convex subset of $H$.

Let $T: C \rightarrow H$ be a nonlinear mapping; we denote the set of fixed points of $T$ by $\operatorname{Fix}(T)$ (i.e., $\operatorname{Fix}(T)=\{x \in C$ : $T x=x\}$ ). A mapping $T: C \rightarrow H$ is called $k$-Lipschitzian continuous if there exists a constant $k>0$ such that

$$
\|T x-T y\| \leq k\|x-y\|, \quad \forall x, y \in C .
$$

In particular, $T$ is said to be a nonexpansive mapping if $k=$ 1. A mapping $B$ is called $\eta$-strongly monotone on $C$, if there exists a constant $\eta>0$ such that

$$
\langle B x-B y, x-y\rangle \geq \eta\|x-y\|^{2}, \quad \forall x, y \in C .
$$

A variational inequality (short for VI) is formulated as finding a point $x^{*} \in C$ such that

$$
\left\langle B x^{*}, x-x^{*}\right\rangle \geq 0, \quad \forall x \in C .
$$

If $B$ is a monotone operator, then VI (3) is known as a monotone variational inequality. If the set $C$ is replaced by the set of $\operatorname{Fix}(T)$ of fixed points of a mapping $T$, then the VI (3) is called a hierarchical variational inequality problem.

Many practical problems in applied sciences such as signal processing [1], beamforming [2], and power control [3] are formulated as the monotone variational inequality with a fixed point constrained. In recent years, several authors paid attention toward this kind of problem. Some methods have been proposed to solve the hierarchical fixed point problems and variational inequalities; see for instance [4-10] and the references therein.

In 2010, Tian [11] proposed a general iterative method and revealed the inner contact of the Yamada's algorithm [12] and viscosity iterative algorithm; then he obtained the following result in a real Hilbert space.

Theorem 1. Let $x_{n}$ be generated by algorithm $x_{n+1}=$ $\alpha_{n} \gamma f\left(x_{n}\right)+\left(I-\alpha_{n} \mu A\right) T x_{n}$ with the sequence $\left\{\alpha_{n}\right\}$ of parameters satisfying conditions $(\mathrm{Cl})-(\mathrm{C} 3)$ :

$$
\begin{aligned}
& \text { (C1) } \alpha_{n} \rightarrow 0, \\
& \text { (C2) } \sum_{n=0}^{\infty} \alpha_{n}=\infty, \\
& \text { (C3) either } \sum_{n=1}^{\infty}\left|\alpha_{n+1}-\alpha_{n}\right|<\infty \text { or } \lim _{n \rightarrow \infty}\left(\alpha_{n+1} / \alpha_{n}\right)=1 \text {. }
\end{aligned}
$$

Then $x_{n}$ converges strongly to a fixed point $\tilde{x}$ of $T$ which solves the variational inequality

$$
\langle(\mu A-\gamma f) \tilde{x}, \tilde{x}-z\rangle \leq 0, \quad \forall z \in \operatorname{Fix}(T) .
$$


Recently, Yao et al. [10] investigated an iterative method for solving a hierarchical fixed point problem by

$$
\begin{gathered}
y_{n}=\beta_{n} S x_{n}+\left(1-\beta_{n}\right) x_{n}, \\
x_{n+1}=P_{C}\left(\alpha_{n} f\left(x_{n}\right)+\left(1-\alpha_{n}\right) T y_{n}\right),
\end{gathered}
$$

where $S, T$ are nonexpansive mapping with $\operatorname{Fix}(T) \neq \emptyset$ and $f$ is a contraction; the sequence converges strongly to the unique solution of the variational inequality

$$
x^{*} \in \operatorname{Fix}(T), \quad\left\langle(I-f) x^{*}, p-x^{*}\right\rangle \leq 0, \quad \forall p \in \operatorname{Fix}(T) .
$$

Very recently, on this basis, Wang and $\mathrm{Xu}$ [8] introduced a new modified iterative method for solving a hierarchical fixed point problem. To be more precise, they proposed the following algorithm:

$$
\begin{gathered}
y_{n}=\beta_{n} S x_{n}+\left(1-\beta_{n}\right) x_{n}, \\
x_{n+1}=P_{C}\left(\alpha_{n} \rho U x_{n}+\left(I-\alpha_{n} \mu F\right) T y_{n}\right),
\end{gathered}
$$

where $S, T$ are nonexpansive mappings with $\operatorname{Fix}(T) \neq \emptyset, U$ is a Lipschitzian mapping, and $F$ is a Lipschitzian and strongly monotone operator. They proved the sequence generated by the above algorithm converges strongly to the unique solution of the variational inequality

$$
\left\langle(\rho U-\mu F) x^{*}, p-x^{*}\right\rangle \leq 0, \quad \forall p \in \operatorname{Fix}(T) .
$$

On the other hand, Tian and Di [13] established a synchronal algorithm and a cyclic algorithm for fixed point problems and variational inequalities. In 2012, Ceng et al. [4] proposed an iterative method to solve a special form of VI (3), where the constraint set is the set of common fixed points of $N$ nonexpansive mappings $T_{1}, T_{2}, \ldots, T_{N}$.

Motivated and inspired by the above works, in this paper, we combine the hybrid steepest descent algorithm and hierarchical variational inequalities to propose a synchronal algorithm and a cyclic algorithm involving finite family of nonexpansive mappings. Under certain assumptions, we will prove that the sequences converge strongly. Further an example will be given to demonstrate the effectiveness of our iterative schemes.

\section{Preliminaries}

Recall that given a nonempty, closed and convex subset $C$ of a real Hilbert space $H$, for any $x \in H$, there exists a unique nearest point in $C$, denoted by $P_{C} x$, such that

$$
\left\|x-P_{C} x\right\| \leq\|x-y\|
$$

for all $y \in C$. Such a $P_{C}$ is called the metric (or the nearest point) projection of $H$ onto $C$. As we all know, $y=P_{C} x$ if and only if there holds the relation

$$
\langle x-y, y-z\rangle \geq 0, \quad \forall z \in C .
$$

In the sequel, we will make use of the following lemmas in a real Hilbert space $H$.
Lemma 2. Let $H$ be a real Hilbert space; the following inequalities hold:

(i) $\|x+y\|^{2} \leq\|x\|^{2}+2\langle y, x+y\rangle, \forall x, y \in H$,

(ii) $\|t x+(1-t) y\|^{2} \leq t\|x\|^{2}+(1-t)\|y\|^{2}, \forall t \in[0,1]$, $\forall x, y \in H$.

Lemma 3 (see [13]). Let $B: H \rightarrow H$ be a $k$-Lipschitzian and $\eta$-strongly monotone operator on a Hilbert space $H$ with $k>0$, $\eta>0,0<\mu<2 \eta / k^{2}$, and $0<t<1$. Then $S=(I-t \mu B)$ : $H \rightarrow H$ is a contraction with contractive coefficient $1-t \tau$ and $\tau=(1 / 2) \mu\left(2 \eta-\mu k^{2}\right)$.

Lemma 4 (see [5]). Let $V: C \rightarrow H$ be an l-Lipschitz mapping with coefficient $l \geq 0$ and $B: C \rightarrow H$ a k-Lipschitzian continuous operator and $\eta$-strongly monotone operator with $k>0, \eta>0$. Then for $0<\gamma<\mu \eta / l$,

$$
\begin{array}{r}
\langle x-y,(\mu B-\gamma V) x-(\mu B-\gamma V) y\rangle \\
\geq(\mu \eta-\gamma l)\|x-y\|^{2}, \quad x, y \in H .
\end{array}
$$

That is, $\mu B-\gamma V$ is strongly monotone with coefficient $\mu \eta-\gamma l$.

Lemma 5 (see [9]). Assume that $\left\{a_{n}\right\}$ is a sequence of nonnegative real numbers such that

$$
a_{n+1} \leq\left(1-\gamma_{n}\right) a_{n}+\delta_{n}
$$

where $\left\{\gamma_{n}\right\}$ is a sequence in $(0,1)$ and $\left\{\delta_{n}\right\}$ is a sequence such that

(i) $\sum_{n=1}^{\infty} \gamma_{n}=\infty$,

(ii) $\lim \sup _{n \rightarrow \infty}\left(\delta_{n} / \gamma_{n}\right) \leq 0$ or $\sum_{n=1}^{\infty}\left|\delta_{n}\right|<\infty$.

Then, $\lim _{n \rightarrow \infty} a_{n}=0$.

Lemma 6 (see [14]). Let $H$ be a real Hilbert, and let $T_{i}$ : $H \rightarrow H(i=1,2, \ldots)$ be all nonexpansive mappings with $\cap_{i=1}^{\infty} \operatorname{Fix}\left(T_{i}\right) \neq \emptyset$. Let $T=\sum_{i=1}^{\infty} \omega_{i} T_{i}(i=1,2, \ldots)$, where $\left\{\omega_{i}\right\} \subset$ $(0,1)$ such that $\sum_{i=1}^{\infty} \omega_{i}=1$. Then $T$ is a nonexpansive mapping with $\operatorname{Fix}(T)=\cap_{i=1}^{\infty} \operatorname{Fix}\left(T_{i}\right)$.

Lemma 7 (see [13]). Let $H$ be a Hilbert space, and let $C$ be a nonempty closed convex subset of $H$ and $T: C \rightarrow C a$ nonexpansive mapping with $\operatorname{Fix}(T) \neq \emptyset$. If $\left\{x_{n}\right\}$ is a sequence in $C$ weakly converging to $x$ and if $\left\{(I-T) x_{n}\right\}$ converges strongly to $y$, then $(I-T) x=y$.

We adopt the following notations:

(1) $x_{n} \rightarrow x$ stands for the weak convergence of $\left\{x_{n}\right\}$ to $x$,

(2) $x_{n} \rightarrow x$ stands for the strong convergence of $\left\{x_{n}\right\}$ to $x$.

\section{Synchronal Algorithm}

Throughout the rest of this paper, we always assume that $V: C \rightarrow H$ is an $l$-Lipschitzian mapping with coefficient $l \geq 0$ and $B: C \rightarrow H$ is a $k$-Lipschitzian continuous operator 
and $\eta$-strongly monotone with $k>0, \eta>0$. Let $N \geq 1$ be an integer. Let, for each $1 \leq i \leq N, T_{i}: C \rightarrow C$ be a nonexpansive mapping and $S: C \rightarrow C$ also nonexpansive. Assume that $0<\mu<2 \eta / k^{2}$ and $0<\gamma<\mu\left(\eta-\mu k^{2} / 2\right) / \alpha=\tau / l$.

Define a mapping $U_{n}=\beta_{n} S+\left(1-\beta_{n}\right) I$. Since $S$ is nonexpansive, it is easy to get that $U_{n}$ is also nonexpansive. Consider the following mapping $G_{n}$ on $C$ defined by

$$
\begin{array}{r}
G_{n} x=P_{C}\left(\alpha_{n} \gamma V(x)+\left(I-\alpha_{n} \mu B\right) T U_{n} x\right), \\
\forall x \in C, n \in \mathbb{N},
\end{array}
$$

where $\alpha_{n} \in(0,1), T=\sum_{i=1}^{N} \omega_{i} T_{i}$ with $\omega_{i}>0$, and $\sum_{i=1}^{N} \omega_{i}=1$. By Lemmas 2 and 3, we obtain

$$
\begin{aligned}
\left\|G_{n} x-G_{n} y\right\| \leq & \alpha_{n} \gamma\|V x-V y\| \\
& +\left(1-\alpha_{n} \tau\right)\left\|T U_{n} x-T U_{n} y\right\| \\
\leq & \alpha_{n} \gamma l\|x-y\|+\left(1-\alpha_{n} \tau\right)\|x-y\| \\
= & \left(1-\alpha_{n}(\tau-\gamma l)\right)\|x-y\| .
\end{aligned}
$$

Since $0<1-\alpha_{n}(\tau-\gamma l)<1$, it follows that $G_{n}$ is a contraction. Therefore, by the Banach contraction principle, $G_{n}$ has a unique fixed point $x_{n}^{V} \in C$ such that

$$
x_{n}^{V}=P_{C}\left(\alpha_{n} \gamma V\left(x_{n}^{V}\right)+\left(I-\alpha_{n} \mu B\right) T U_{n} x_{n}^{V}\right) .
$$

For simplicity, we will write $x_{n}$ for $x_{n}^{V}$ provided that no confusion occurs. Next we prove that the sequence $\left\{x_{n}\right\}$ converges strongly to a point $x^{*} \in \Omega=\cap_{i=1}^{N} \operatorname{Fix}\left(T_{i}\right)$ which solves the variational inequality

$$
\left\langle(\gamma V-\mu B) x^{*}, p-x^{*}\right\rangle \leq 0, \quad \forall p \in \Omega .
$$

By the property of the projection, we can get $x^{*}=P_{\Omega}(I-\mu B+$ $\gamma V) x^{*}$ equivalently.

Theorem 8. Let $C$ be a nonempty, closed, and convex subset of a real Hilbert space $H$ and $V: C \rightarrow H$ an l-Lipschitzian mapping with $l \geq 0$. Let $N \geq 1$ be an integer. Let, for each $1 \leq i \leq N, T_{i}: C \rightarrow C$ be a nonexpansive mapping and let $S: C \rightarrow C$ be also nonexpansive. Assume the set $\Omega=\bigcap_{i=1}^{N} \operatorname{Fix}\left(T_{i}\right) \neq \emptyset$. Let $B: C \rightarrow H$ be a $k$-Lipschitzian continuous operator and $\eta$-strongly monotone with $k>0$, $\eta>0,0<\mu<2 \eta / k^{2}$ and $0<\gamma<\mu\left(\eta-\mu k^{2} / 2\right) / l=\tau / l$. Given $x_{1} \in C$, let $\left\{x_{n}\right\}$ be the sequence generated by the following algorithm:

$$
\begin{gathered}
y_{n}=\beta_{n} S x_{n}+\left(1-\beta_{n}\right) x_{n}, \\
x_{n+1}=P_{C}\left(\alpha_{n} \gamma V x_{n}+\left(I-\mu \alpha_{n} B\right) T y_{n}\right) .
\end{gathered}
$$

If $\left\{\alpha_{n}\right\}$ and $\left\{\beta_{n}\right\}$ satisfy the following properties:

(i) $\left\{\alpha_{n}\right\} \subset(0,1), \lim _{n \rightarrow \infty} \alpha_{n}=0$ and $\sum_{n=1}^{\infty} \alpha_{n}=\infty$,

(ii) $\left\{\beta_{n}\right\} \subset[0,1), \lim _{n \rightarrow \infty}\left(\beta_{n} / \alpha_{n}\right)=0$,

(iii) $\sum_{n=1}^{\infty}\left|\alpha_{n+1}-\alpha_{n}\right|<\infty$ and $\sum_{n=1}^{\infty}\left|\beta_{n+1}-\beta_{n}\right|<\infty$.

Then, $\left\{x_{n}\right\}$ converges strongly to $x^{*} \in \Omega$, which solves the variational inequality (16).
Proof. The proof is divided into several steps.

Step 1. Show first that $\left\{x_{n}\right\}$ is bounded.

Take any $p \in \Omega$; we have

$$
\begin{aligned}
\left\|y_{n}-p\right\|= & \left\|\beta_{n}\left(S x_{n}-p\right)+\left(1-\beta_{n}\right)\left(x_{n}-p\right)\right\| \\
\leq & \beta_{n}\left\|S x_{n}-S p\right\|+\beta_{n}\|S p-p\| \\
& +\left(1-\beta_{n}\right)\left\|x_{n}-p\right\| \leq\left\|x_{n}-p\right\|+\beta_{n}\|S p-p\| .
\end{aligned}
$$

Further we get

$$
\begin{aligned}
\| x_{n+1}- & p \| \\
= & \left\|P_{C}\left(\alpha_{n} \gamma V x_{n}+\left(I-\mu \alpha_{n} B\right) T y_{n}\right)-P_{C} p\right\| \\
\leq & \| \alpha_{n}\left(\gamma V x_{n}-\mu B p\right) \\
& +\left(I-\mu \alpha_{n} B\right) T y_{n}-\left(I-\mu \alpha_{n} B\right) p \| \\
\leq & \alpha_{n}\left(\left\|\gamma V x_{n}-\gamma V p\right\|+\|\gamma V p-\mu B p\|\right) \\
& +\left(1-\alpha_{n} \tau\right)\left\|T y_{n}-p\right\| \\
\leq & \alpha_{n} l \gamma\left\|x_{n}-p\right\|+\alpha_{n}\|\gamma V p-\mu B p\| \\
& +\left(1-\alpha_{n} \tau\right)\left\|y_{n}-p\right\| \\
= & \left(1-\alpha_{n}(\tau-l \gamma)\right)\left\|x_{n}-p\right\| \\
& +\alpha_{n}(\tau-l \gamma) \frac{\|\gamma V p-\mu B p\|+\|S p-p\|}{\tau-l \gamma} \\
\leq & \max \left\{\left\|x_{n}-p\right\|, \frac{\|\gamma V p-\mu B p\|+\|S p-p\|}{\tau-l \gamma}\right\} .
\end{aligned}
$$

By induction, we obtain $\left\|x_{n}-p\right\| \leq \max \left\{\left\|x_{1}-p\right\|,(\| \gamma V p-\right.$ $\mu B p\|+\| S p-p \|) /(\tau-l \gamma)\}, n \geq 1$. Hence, $\left\{x_{n}\right\}$ is bounded, so is $\left\{y_{n}\right\}$. It follows from the Lipschitz continuity of $B$ and $V$ that $\left\{B x_{n}\right\},\left\{B u_{n}\right\}$, and $\left\{V x_{n}\right\}$ are also bounded. From the nonexpansivity of $T$ and $S$, it follows that $\left\{T x_{n}\right\},\left\{S x_{n}\right\}$, and $\left\{B T y_{n}\right\}$ are also bounded.

Step 2. Show that

$$
\lim _{n \rightarrow \infty}\left\|x_{n+1}-x_{n}\right\|=0
$$

By (17), we have

$$
\begin{aligned}
& \left\|x_{n+1}-x_{n}\right\| \\
& =\| P_{C}\left(\alpha_{n} \gamma V x_{n}+\left(I-\mu \alpha_{n} B\right) T y_{n}\right) \\
& \quad-P_{C}\left(\alpha_{n-1} \gamma V x_{n-1}+\left(I-\mu \alpha_{n-1} B\right) T y_{n-1}\right) \| \\
& \leq \| \alpha_{n} \gamma V x_{n}+\left(I-\mu \alpha_{n} B\right) T y_{n} \\
& \quad-\left(\alpha_{n-1} \gamma V x_{n-1}+\left(I-\mu \alpha_{n-1} B\right) T y_{n-1}\right) \| \\
& \leq \alpha_{n} \gamma\left\|V x_{n}-V x_{n-1}\right\| \\
& +\left\|\left(I-\mu \alpha_{n} B\right) T y_{n}-\left(I-\mu \alpha_{n} B\right) T y_{n-1}\right\| \\
& +\left\|\left(I-\mu \alpha_{n} B\right) T y_{n-1}-\left(I-\mu \alpha_{n-1} B\right) T y_{n-1}\right\| \\
& +\left|\alpha_{n}-\alpha_{n-1}\right| \gamma\left\|V x_{n-1}\right\|
\end{aligned}
$$




$$
\begin{aligned}
\leq & \alpha_{n} \gamma l\left\|x_{n}-x_{n-1}\right\| \\
& +\left|\alpha_{n}-\alpha_{n-1}\right|\left(\gamma l\left\|V x_{n-1}\right\|+\mu\left\|B T y_{n-1}\right\|\right) \\
& +\left(1-\alpha_{n} \tau\right)\left\|y_{n}-y_{n-1}\right\| .
\end{aligned}
$$

Observe that

$$
\begin{aligned}
\| y_{n}- & y_{n-1} \| \\
= & \| \beta_{n} S x_{n}+\left(1-\beta_{n}\right) x_{n}-\beta_{n-1} S x_{n-1} \\
& \quad-\left(1-\beta_{n-1}\right) x_{n-1} \| \\
\leq & \left\|\beta_{n} S x_{n}-\beta_{n} S x_{n-1}\right\| \\
& +\left\|\left(1-\beta_{n}\right) x_{n}-\left(1-\beta_{n}\right) x_{n-1}\right\| \\
& +\left\|\beta_{n} S x_{n-1}-\beta_{n-1} S x_{n-1}\right\| \\
& +\left\|\left(1-\beta_{n}\right) x_{n-1}-\left(1-\beta_{n-1}\right) x_{n-1}\right\| \\
\leq & \beta_{n}\left\|x_{n}-x_{n-1}\right\|+\left(1-\beta_{n}\right)\left\|x_{n}-x_{n-1}\right\| \\
& +\left|\beta_{n}-\beta_{n-1}\right|\left\|S x_{n-1}\right\|+\left|\beta_{n}-\beta_{n-1}\right|\left\|x_{n-1}\right\| \\
= & \left\|x_{n}-x_{n-1}\right\| \\
& +\left|\beta_{n}-\beta_{n-1}\right|\left(\left\|S x_{n-1}\right\|+\left\|x_{n-1}\right\|\right) .
\end{aligned}
$$

Together with (21) and (22), we get

$$
\begin{aligned}
\| x_{n+1} & -x_{n} \| \\
\leq & \alpha_{n} \gamma l\left\|x_{n}-x_{n-1}\right\| \\
& +\left|\alpha_{n}-\alpha_{n-1}\right|\left(\gamma l\left\|V x_{n-1}\right\|+\mu\left\|B T y_{n-1}\right\|\right) \\
& +\left(1-\alpha_{n} \tau\right)\left\|x_{n}-x_{n-1}\right\| \\
& +\left|\beta_{n}-\beta_{n-1}\right|\left(\left\|S x_{n-1}\right\|+\left\|x_{n-1}\right\|\right) \\
= & \left(1-\alpha_{n}(\tau-\gamma l)\right)\left\|x_{n}-x_{n-1}\right\| \\
& +\left|\alpha_{n}-\alpha_{n-1}\right|\left(\gamma l\left\|V x_{n-1}\right\|+\mu\left\|B T y_{n-1}\right\|\right) \\
& +\left|\beta_{n}-\beta_{n-1}\right|\left(\left\|S x_{n-1}\right\|+\left\|x_{n-1}\right\|\right) \\
\leq & \left(1-\alpha_{n}(\tau-\gamma l)\right)\left\|x_{n}-x_{n-1}\right\| \\
& +\left(\left|\alpha_{n}-\alpha_{n-1}\right|+\left|\beta_{n}-\beta_{n-1}\right|\right) M_{1},
\end{aligned}
$$

where $M_{1}=\sup _{n}\left\{\left\|\gamma l V x_{n-1}\right\|+\mu\left\|B T y_{n-1}\right\|+\left\|S x_{n-1}\right\|+\left\|x_{n-1}\right\|\right\}$. By Lemma 5, we obtain

$$
\left\|x_{n+1}-x_{n}\right\| \longrightarrow 0
$$

Step 3. Show that

$$
\left\|x_{n}-T x_{n}\right\| \longrightarrow 0
$$

Observe that

$$
\begin{aligned}
& \left\|x_{n+1}-T y_{n}\right\| \\
& \quad=\left\|P_{C}\left(\alpha_{n} \gamma V x_{n}+\left(I-\mu \alpha_{n} B T y_{n}\right)\right)-P_{C} T y_{n}\right\| \\
& \quad \leq \alpha_{n}\left\|\gamma V x_{n}-\mu B T y_{n}\right\| \longrightarrow 0 .
\end{aligned}
$$

From condition (i) and (ii), we obtain

$$
\begin{aligned}
& \left\|x_{n+1}-T x_{n}\right\| \\
& \quad \leq\left\|x_{n+1}-T y_{n}\right\|+\left\|T y_{n}-T x_{n}\right\| \\
& \quad \leq\left\|x_{n+1}-T y_{n}\right\|+\left\|y_{n}-x_{n}\right\| \\
& \quad=\left\|x_{n+1}-T y_{n}\right\|+\beta_{n}\left\|S x_{n}-x_{n}\right\| \longrightarrow 0 .
\end{aligned}
$$

Step 4. Show that

$$
\limsup _{n \rightarrow \infty}\left\langle(\gamma V-\mu B) x^{*}, x_{n}-x^{*}\right\rangle \leq 0,
$$

where $x^{*}=P_{\Omega}(I-\mu B+\gamma V) x^{*}$ is a unique solution of the variational inequality (16).

Indeed, take a subsequence $\left\{x_{n_{j}}\right\}$ of $\left\{x_{n}\right\}$ such that

$$
\begin{aligned}
\limsup _{n \rightarrow \infty} & \left\langle(\gamma V-\mu B) x^{*}, x_{n}-x^{*}\right\rangle \\
& =\lim _{j \rightarrow \infty}\left\langle(\gamma V-\mu B) x^{*}, x_{n_{j}}-x^{*}\right\rangle .
\end{aligned}
$$

Since $\left\{x_{n_{j}}\right\}$ is bounded, there exists a subsequence $\left\{x_{n_{j_{k}}}\right\}$ of $\left\{x_{n_{j}}\right\}$ which converges weakly to $\hat{x}$. Without loss of generality, we can assume $x_{n_{j}} \rightarrow \widehat{x}$ and

$$
\left\|x_{n_{j}}-T x_{n_{j}}\right\| \longrightarrow 0
$$

By Lemma 7, we have $\widehat{x}=T \widehat{x}$. From Lemma 6 , we get

$$
\widehat{x} \in \operatorname{Fix}(T)=\bigcap_{i=1}^{N} \operatorname{Fix}\left(T_{i}\right) .
$$

Since $x^{*}=P_{\Omega}(I-\mu B+\gamma V) x^{*}$, it follows that

$$
\begin{aligned}
& \limsup _{n \rightarrow \infty}\left\langle(\gamma V-\mu B) x^{*}, x_{n}-x^{*}\right\rangle \\
& \quad=\lim _{j \rightarrow \infty}\left\langle(\gamma V-\mu B) x^{*}, x_{n_{j}}-x^{*}\right\rangle \\
& =\left\langle(\gamma V-\mu B) x^{*}, \widehat{x}-x^{*}\right\rangle \leq 0 .
\end{aligned}
$$

Step 5. Show that

$$
x_{n} \longrightarrow x^{*} \text {. }
$$

Denote $z_{n}=\alpha_{n} \gamma V x_{n}+\left(I-\mu \alpha_{n} B\right) T y_{n}$, then $x_{n+1}=P_{C} z_{n}$. From (17), we have

$$
\begin{aligned}
& \left\|x_{n+1}-x^{*}\right\|^{2} \\
& =\left\|P_{C} z_{n}-x^{*}\right\|^{2}=\left\langle P_{C} z_{n}-z_{n}, P_{C} z_{n}-x^{*}\right\rangle \\
& \quad+\left\langle z_{n}-x^{*}, x_{n+1}-x^{*}\right\rangle \leq\left\langle z_{n}-x^{*}, x_{n+1}-x^{*}\right\rangle
\end{aligned}
$$




$$
\begin{aligned}
= & \left\langle\left(I-\mu \alpha_{n} B\right) T y_{n}-\left(I-\mu \alpha_{n} B\right) T x^{*}\right. \\
& \left.+\alpha_{n}\left(\gamma V x_{n}-\mu B x^{*}\right), x_{n+1}-x^{*}\right\rangle \\
\leq & \left(1-\alpha_{n} \tau\right)\left\|y_{n}-x^{*}\right\|\left\|x_{n+1}-x^{*}\right\| \\
& +\alpha_{n}\left\langle\gamma V x_{n}-\gamma V x^{*}, x_{n+1}-x^{*}\right\rangle \\
& +\alpha_{n}\left\langle\gamma V x^{*}-\mu B x^{*}, x_{n+1}-x^{*}\right\rangle \\
\leq & \left(1-\alpha_{n} \tau\right)\left(\left\|x_{n}-x^{*}\right\|+\beta_{n}\left\|S x^{*}-x^{*}\right\|\right)\left\|x_{n+1}-x^{*}\right\| \\
& +\alpha_{n} \gamma l\left\|x_{n}-x^{*}\right\|\left\|x_{n+1}-x^{*}\right\| \\
& +\alpha_{n}\left\langle\gamma V x^{*}-\mu B x^{*}, x_{n+1}-x^{*}\right\rangle \\
\leq & \frac{1-\alpha_{n}(\tau-\gamma l)}{2}\left(\left\|x_{n}-x^{*}\right\|^{2}+\left\|x_{n+1}-x^{*}\right\|^{2}\right) \\
& +\beta_{n}\left\|x_{n+1}-x^{*}\right\|\left\|S x^{*}-x^{*}\right\| \\
& +\alpha_{n}\left\langle\gamma V x^{*}-\mu B x^{*}, x_{n+1}-x^{*}\right\rangle .
\end{aligned}
$$

This implies that

$$
\begin{aligned}
\left\|x_{n+1}-x^{*}\right\|^{2} \\
\leq \frac{1-\alpha_{n}(\tau-\gamma l)}{1+\alpha_{n}(\tau-l \gamma)}\left\|x_{n}-x^{*}\right\|^{2}+\frac{2}{1+\alpha_{n}(\tau-\gamma l)} \\
\times\left(\beta_{n}\left\|x_{n+1}-x^{*}\right\|\left\|S x^{*}-x^{*}\right\|\right. \\
\left.\quad+\alpha_{n}\left\langle\gamma V x^{*}-\mu B x^{*}, x_{n+1}-x^{*}\right\rangle\right) \\
\leq\left(1-\alpha_{n}(\tau-\gamma l)\right)\left\|x_{n}-x^{*}\right\|^{2} \\
+2 \beta_{n} M_{2}+2 \alpha_{n}\left\langle\gamma V x^{*}-\mu B x^{*}, x_{n+1}-x^{*}\right\rangle,
\end{aligned}
$$

where $M_{2}=\sup _{n}\left\|x_{n+1}-x^{*}\right\|\left\|S x^{*}-x^{*}\right\|, n \geq 1$. Put $\gamma_{n}=$ $\alpha_{n}(\tau-l \gamma), \delta_{n}=2 \beta_{n} M_{2}+2 \alpha_{n}\left\langle\gamma V x^{*}-\mu B x^{*}, x_{n+1}-x^{*}\right\rangle$. It is easy to see that $\lim _{\sup _{n \rightarrow \infty}} \delta_{n} / \gamma_{n} \leq 0$. Hence by Lemma 5 , the sequence $\left\{x_{n}\right\}$ converges strongly to $x^{*}$.

Remark 9. Let $N=1$ in Theorem 8; we can get Theorem 3.1 of [8].

Remark 10. Let $N=1, \gamma=1, \mu=1, B=I$ and $V$, be a contraction in Theorem 8 ; it is easy to get the theorem of [10].

\section{Cyclic Algorithm}

In this section, we consider the cyclic algorithm of $N$ nonexpansive mappings $T_{1}, T_{2}, \ldots, T_{N}$. Similarly, we can get that the mapping $G_{n}$ on $C$ defined by

$$
\begin{array}{r}
G_{n} x=P_{C}\left(\alpha_{n} \gamma V(x)+\left(I-\alpha_{n} \mu B\right) T_{[n]} U_{n} x\right), \\
\forall x \in C, n \in \mathbb{N},
\end{array}
$$

is a contraction, where $T_{[n]}=T_{i}$ with $i=n(\bmod ) N$ taking values in $\{1,2, \ldots, N\}$.
Theorem 11. Let $C$ be a nonempty, closed, and convex subset of a real Hilbert space $H$, and let $V: C \rightarrow H$ be an $l$ Lipschitzian mapping with $l \geq 0$. Let $N \geq 1$ be an integer. Let, for each $1 \leq i \leq N, T_{i}: C \rightarrow C$ be a nonexpansive mapping and let $S: C \rightarrow C$ be also nonexpansive. Assume the set $\Omega=\bigcap_{i=1}^{N} \operatorname{Fix}\left(T_{i}\right) \neq \emptyset$. Let $B: C \rightarrow H$ be a $k$-Lipschitzian continuous operator and $\eta$-strongly monotone with $k>0$, $\eta>0,0<\mu<2 \eta / k^{2}$, and $0<\gamma<\mu\left(\eta-\mu k^{2} / 2\right) / l=\tau / l$. Given $x_{1} \in C$, let $\left\{x_{n}\right\}$ be the sequence generated by the following algorithm:

$$
\begin{gathered}
y_{n}=\beta_{n} S x_{n}+\left(1-\beta_{n}\right) x_{n}, \\
x_{n+1}=P_{C}\left(\alpha_{n} \gamma V x_{n}+\left(I-\mu \alpha_{n} B\right) T_{[n]} y_{n}\right) .
\end{gathered}
$$

If $\left\{\alpha_{n}\right\}$ and $\left\{\beta_{n}\right\}$ satisfy the following properties:

(i) $\left\{\alpha_{n}\right\} \subset(0,1), \lim _{n \rightarrow \infty} \alpha_{n}=0$ and $\sum_{n=1}^{\infty} \alpha_{n}=\infty$,

(ii) $\left\{\beta_{n}\right\} \subset[0,1), \lim _{n \rightarrow \infty}\left(\beta_{n} / \alpha_{n}\right)=0$,

(iii) $\sum_{n=1}^{\infty}\left|\alpha_{n+1}-\alpha_{n}\right|<\infty$ and $\sum_{n=1}^{\infty}\left|\beta_{n+1}-\beta_{n}\right|<\infty$.

Assume in addition that

$$
\begin{aligned}
\bigcap_{i=1}^{N} \operatorname{Fix}\left(T_{i}\right) & =\operatorname{Fix}\left(T_{N} T_{N-1} \cdots T_{2} T_{1}\right) \\
& =\operatorname{Fix}\left(T_{1} T_{N} T_{N-1} \cdots T_{2}\right) \\
& =\cdots=\operatorname{Fix}\left(T_{2} T_{1} T_{N} \cdots T_{3}\right) .
\end{aligned}
$$

Then, $\left\{x_{n}\right\}$ converges strongly to $x^{*} \in \Omega$, which solves the variational inequality (16).

Proof. The proof is divided into several steps.

Step 1. Show first that $\left\{x_{n}\right\}$ is bounded.

The proof of Step 1 is similar to that of Theorem 8 .

Step 2. Show that

$$
\lim _{n \rightarrow \infty}\left\|x_{n+N}-x_{n}\right\|=0
$$

By (37), we have

$$
\begin{aligned}
& \left\|x_{n+N+1}-x_{n+1}\right\| \\
& \leq \| \alpha_{n+N} \gamma V x_{n+N}+\left(I-\mu \alpha_{n+N} B\right) T_{[n]} y_{n+N} \\
& \quad-\alpha_{n} \gamma V x_{n}-\left(I-\mu \alpha_{n} B\right) T_{[n]} y_{n} \| \\
& \leq \alpha_{n+N} \gamma\left\|V x_{n+N}-V x_{n}\right\| \\
& +\|\left(I-\mu \alpha_{n+N} B\right) T_{[n]} y_{n+N} \\
& \quad-\left(I-\mu \alpha_{n+N} B\right) T_{[n]} y_{n} \| \\
& +\|\left(I-\mu \alpha_{n+N} B\right) T_{[n]} y_{n} \\
& \quad-\left(I-\mu \alpha_{n} B\right) T_{[n]} y_{n} \| \\
& +\left|\alpha_{n+N}-\alpha_{n}\right| \gamma\left\|V x_{n}\right\|
\end{aligned}
$$




$$
\begin{aligned}
\leq & \alpha_{n+N} \gamma l\left\|x_{n+N}-x_{n}\right\| \\
& +\left(1-\alpha_{n+N} \tau\right)\left\|y_{n+N}-y_{n}\right\| \\
& +\left|\alpha_{n+N}-\alpha_{n}\right|\left(\gamma l\left\|V x_{n}\right\|+\mu\left\|B T_{[n]} y_{n}\right\|\right) .
\end{aligned}
$$

Observe that

$$
\begin{aligned}
\left\|y_{n+N}-y_{n}\right\| & \\
= & \| \beta_{n+N} S x_{n+N}+\left(1-\beta_{n+N}\right) x_{n+N} \\
& \quad-\beta_{n} S x_{n}-\left(1-\beta_{n}\right) x_{n} \| \\
\leq & \left\|\beta_{n+N} S x_{n+N}-\beta_{n+N} S x_{n}\right\| \\
& +\left\|\left(1-\beta_{n+N}\right) x_{n+N}-\left(1-\beta_{n+N}\right) x_{n}\right\| \\
& +\left\|\beta_{n+N} S x_{n}-\beta_{n} S x_{n}\right\| \\
& +\left\|\left(1-\beta_{n+N}\right) x_{n}-\left(1-\beta_{n}\right) x_{n}\right\| \\
\leq & \beta_{n+N}\left\|x_{n+N}-x_{n}\right\| \\
& +\left(1-\beta_{n+N}\right)\left\|x_{n+N}-x_{n}\right\| \\
& +\left|\beta_{n+N}-\beta_{n}\right|\left\|S x_{n}\right\|+\left|\beta_{n+N}-\beta_{n}\right|\left\|x_{n}\right\| \\
= & \left\|x_{n+N}-x_{n}\right\| \\
& +\left|\beta_{n+N}-\beta_{n}\right|\left(\left\|S x_{n}\right\|+\left\|x_{n}\right\|\right) .
\end{aligned}
$$

Together with (40) and (41), we have

$$
\begin{aligned}
&\left\|x_{n+N+1}-x_{n+1}\right\| \\
& \leq \alpha_{n+N} \gamma l\left\|x_{n+N}-x_{n}\right\| \\
&+\left|\alpha_{n+N}-\alpha_{n}\right|\left(\gamma l\left\|V x_{n}\right\|+\mu\left\|B T_{[n]} y_{n}\right\|\right) \\
&+\left(1-\alpha_{n+N} \tau\right)\left\|x_{n+N}-x_{n}\right\| \\
&+\left|\beta_{n+N}-\beta_{n}\right|\left(\left\|S x_{n}\right\|+\left\|x_{n}\right\|\right) \\
&=\left(1-\alpha_{n+N}(\tau-\gamma l)\right)\left\|x_{n+N}-x_{n}\right\| \\
&+\left|\alpha_{n+N}-\alpha_{n}\right|\left(\gamma l\left\|V x_{n}\right\|+\mu\left\|B T_{[n]} y_{n}\right\|\right) \\
&+\left|\beta_{n+N}-\beta_{n}\right|\left(\left\|S x_{n}\right\|+\left\|x_{n}\right\|\right) \\
& \leq\left(1-\alpha_{n+N}(\tau-\gamma l)\right)\left\|x_{n+N}-x_{n}\right\| \\
&+\left(\left|\alpha_{n+N}-\alpha_{n}\right|+\left|\beta_{n+N}-\beta_{n}\right|\right) M_{3},
\end{aligned}
$$

where $M_{3}=\sup _{n}\left\{\left\|\gamma l V x_{n}\right\|+\mu\left\|B T_{[n]} y_{n}\right\|+\left\|S x_{n}\right\|+\left\|x_{n}\right\|\right\}$.

By Lemma 5, we get

$$
\left\|x_{n+N}-x_{n}\right\| \longrightarrow 0 .
$$

Step 3. Show that

$$
\left\|x_{n}-T_{[n+N-1]} T_{[n+N-2]} \cdots T_{[n]} x_{n}\right\| \longrightarrow 0 .
$$

Observe that

$$
\begin{aligned}
& \left\|x_{n+1}-T_{[n]} y_{n}\right\| \\
& \quad=\left\|P_{C}\left(\alpha_{n} \gamma V x_{n}+\left(I-\mu \alpha_{n}\right) B T_{[n]} y_{n}\right)-P_{C} T_{[n]} y_{n}\right\| \\
& \quad \leq \alpha_{n}\left\|\gamma V x_{n}-\mu B T_{[n]} y_{n}\right\| \longrightarrow 0 .
\end{aligned}
$$

From conditions (i) and (ii) of Theorem 11, we obtain

$$
\begin{aligned}
& \left\|x_{n+1}-T_{[n]} x_{n}\right\| \\
& \quad \leq\left\|x_{n+1}-T_{[n]} y_{n}\right\|+\left\|T_{[n]} y_{n}-T_{[n]} x_{n}\right\| \\
& \quad \leq\left\|x_{n+1}-T_{[n]} y_{n}\right\|+\left\|y_{n}-x_{n}\right\| \\
& \quad=\left\|x_{n+1}-T_{[n]} y_{n}\right\|+\beta_{n}\left\|S x_{n}-x_{n}\right\| \longrightarrow 0 .
\end{aligned}
$$

Recursively,

$$
\begin{gathered}
\left\|x_{n+N}-T_{[n+N-1]} x_{n+N-1}\right\| \longrightarrow 0, \\
\left\|x_{n+N-1}-T_{[n+N-2]} x_{n+N-2}\right\| \longrightarrow 0 .
\end{gathered}
$$

Since every $T_{[n]}$ is nonexpansive, it is easy to get

$\left\|T_{[n+N-1]} x_{n+N-1}-T_{[n+N-1]} T_{[n+N-2]} x_{n+N-2}\right\| \longrightarrow 0$.

Similarly, we obtain

$$
\begin{aligned}
& \| T_{[n+N-1]} T_{[n+N-2]} x_{n+N-2} \\
& \quad-T_{[n+N-1]} T_{[n+N-2]} T_{[n+N-3]} x_{n+N-3} \| \longrightarrow 0
\end{aligned}
$$

$$
\begin{aligned}
& \| T_{[n+N-1]} T_{[n+N-2]} \cdots T_{[n+1]} x_{n+1} \\
& \quad-T_{[n+N-1]} T_{[n+N-2]} \cdots T_{[n]} x_{n} \| \longrightarrow 0 .
\end{aligned}
$$

Thus we get

$$
\begin{aligned}
& \left\|x_{n+N}-T_{[n+N-1]} \cdots T_{[n]} x_{n}\right\| \\
& \leq\left\|x_{n+N}-T_{[n+N-1]} x_{n+N-1}\right\| \\
& +\| T_{[n+N-1]} x_{n+N-1} \\
& \quad-T_{[n+N-1]} T_{[n+N-2]} x_{n+N-2} \| \\
& +\cdots+\| T_{[n+N-1]} T_{[n+N-2]} \cdots T_{[n+1]} x_{n+1} \\
& \quad-T_{[n+N-1]} \cdots T_{[n]} x_{n} \| \longrightarrow 0 .
\end{aligned}
$$

Since

$$
\begin{aligned}
& \left\|x_{n}-T_{[n+N-1]} T_{[n+N-2]} \cdots T_{[n]} x_{n}\right\| \\
& \quad \leq\left\|x_{n}-x_{n+N}\right\|+\left\|x_{n+N}-T_{[n+N-1]} \cdots T_{[n]} x_{n}\right\|,
\end{aligned}
$$

we obtain (44).

Step 4. Show that

$$
\limsup _{n \rightarrow \infty}\left\langle(\gamma V-\mu B) x^{*}, x_{n}-x^{*}\right\rangle \leq 0,
$$


where $x^{*}=P_{\Omega}(I-\mu B+\gamma V) x^{*}$ is a unique solution of the variational inequality (16).

Indeed, take a subsequence $\left\{x_{n_{j}}\right\}$ of $\left\{x_{n}\right\}$ such that

$$
\begin{aligned}
\limsup _{n \rightarrow \infty} & \left\langle(\gamma V-\mu B) x^{*}, x_{n}-x^{*}\right\rangle \\
= & \lim _{j \rightarrow \infty}\left\langle(\gamma V-\mu B) x^{*}, x_{n_{j}}-x^{*}\right\rangle .
\end{aligned}
$$

Since $\left\{x_{n_{j}}\right\}$ is bounded, there exists a subsequence $\left\{x_{n_{j_{k}}}\right\}$ of $\left\{x_{n_{j}}\right\}$ which converges weakly to $\hat{x}$. Without loss of generality, we can assume $x_{n_{j}} \rightarrow \widehat{x}$ and

$$
\left\|x_{n_{j}}-T_{\left[n_{j}+N-1\right]} T_{\left[n_{j}+N-2\right]} \cdots T_{\left[n_{j}\right]} x_{n_{j}}\right\| \longrightarrow 0 .
$$

Notice that, for each $n_{j}, T_{\left[n_{j}+N-1\right]} T_{\left[n_{j}+N-2\right]} \cdots T_{\left[n_{j}\right]}$ is some permutation of the mappings $T_{1} T_{2} \cdots T_{N}$. Since $T_{1} T_{2} \cdots T_{N}$ are finite, all the finite permutations are $N$ !; there must be some permutation that appears infinite times. Without loss of generality, we can assume this permutation is $T_{N} T_{N-1} \cdots T_{1}$. We obtain

$$
\left\|x_{n_{j}}-T_{N} T_{N-1} \cdots T_{1} x_{n_{j}}\right\| \longrightarrow 0 .
$$

Obviously, $T_{N} T_{N-1} \cdots T_{1}$ is nonexpansive. By Lemma 7, we have $\hat{x}=T_{N} T_{N-1} \cdots T_{1} \widehat{x}$. Further by the assumption in Theorem 11, we get

$$
\widehat{x} \in \operatorname{Fix}\left(T_{N} T_{N-1} \cdots T_{1}\right)=\bigcap_{i=1}^{N} \operatorname{Fix}\left(T_{i}\right) .
$$

Since $x^{*}=P_{\Omega}(I-\mu B+\gamma V) x^{*}$, it follows that

$$
\begin{aligned}
\limsup _{n \rightarrow \infty} & \left\langle(\gamma V-\mu B) x^{*}, x_{n}-x^{*}\right\rangle \\
= & \lim _{j \rightarrow \infty}\left\langle(\gamma V-\mu B) x^{*}, x_{n_{j}}-x^{*}\right\rangle \\
= & \left\langle(\gamma V-\mu B) x^{*}, \widehat{x}-x^{*}\right\rangle \leq 0 .
\end{aligned}
$$

Step 5. Show that

$$
x_{n} \longrightarrow x^{*} \text {. }
$$

Denote $z_{n}=\alpha_{n} \gamma V x_{n}+\left(I-\mu \alpha_{n} B\right) T_{[n]} y_{n}$; then $x_{n+1}=P_{C} z_{n}$. From (37), we have

$$
\begin{aligned}
\left\|x_{n+1}-x^{*}\right\|^{2} \\
=\left\|P_{C} z_{n}-x^{*}\right\|^{2}=\left\langle P_{C} z_{n}-z_{n}, P_{C} z_{n}-x^{*}\right\rangle \\
\quad+\left\langle z_{n}-x^{*}, x_{n+1}-x^{*}\right\rangle \leq\left\langle z_{n}-x^{*}, x_{n+1}-x^{*}\right\rangle \\
=\left\langle\left(I-\mu \alpha_{n} B\right) T_{[n]} y_{n}-\left(I-\mu \alpha_{n} B\right) T_{[n]} x^{*}\right. \\
\left.\quad+\alpha_{n}\left(\gamma V x_{n}-\mu B x^{*}\right), x_{n+1}-x^{*}\right\rangle \\
\leq\left(1-\alpha_{n} \tau\right)\left\|y_{n}-x^{*}\right\|\left\|x_{n+1}-x^{*}\right\| \\
+\alpha_{n}\left\langle\gamma V x_{n}-\gamma V x^{*}, x_{n+1}-x^{*}\right\rangle \\
+\alpha_{n}\left\langle\gamma V x^{*}-\mu B x^{*}, x_{n+1}-x^{*}\right\rangle
\end{aligned}
$$

$$
\begin{aligned}
\leq & \left(1-\alpha_{n} \tau\right)\left(\left\|x_{n}-x^{*}\right\|+\beta_{n}\left\|S x^{*}-x^{*}\right\|\right) \\
& \times\left\|x_{n+1}-x^{*}\right\|+\alpha_{n} \gamma l\left\|x_{n}-x^{*}\right\|\left\|x_{n+1}-x^{*}\right\| \\
& +\alpha_{n}\left\langle\gamma V x^{*}-\mu B x^{*}, x_{n+1}-x^{*}\right\rangle \\
\leq & \frac{1-\alpha_{n}(\tau-\gamma l)}{2}\left(\left\|x_{n}-x^{*}\right\|^{2}+\left\|x_{n+1}-x^{*}\right\|^{2}\right) \\
& +\beta_{n}\left\|x_{n}-x^{*}\right\|\left\|S x^{*}-x^{*}\right\| \\
& +\alpha_{n}\left\langle\gamma V x^{*}-\mu B x^{*}, x_{n+1}-x^{*}\right\rangle .
\end{aligned}
$$

This implies that

$$
\begin{aligned}
& \left\|x_{n+1}-x^{*}\right\|^{2} \\
& \leq \frac{1-\alpha_{n}(\tau-\gamma l)}{1+\alpha_{n}(\tau-\gamma l)}\left\|x_{n}-x^{*}\right\|^{2}+\frac{2}{1+\alpha_{n}(\tau-\gamma l)} \\
& \quad \times\left(\beta_{n}\left\|x_{n}-x^{*}\right\|\left\|S x^{*}-x^{*}\right\|\right. \\
& \left.\quad+\alpha_{n}\left\langle\gamma V x^{*}-\mu B x^{*}, x_{n+1}-x^{*}\right\rangle\right) \\
& \leq\left(1-\alpha_{n}(\tau-\gamma l)\right)\left\|x_{n}-x^{*}\right\|^{2} \\
& \quad+2 \beta_{n} M_{1}+2 \alpha_{n}\left\langle\gamma V x^{*}-\mu B x^{*}, x_{n+1}-x^{*}\right\rangle,
\end{aligned}
$$

where $M_{1}=\sup _{n}\left\|x_{n}-x^{*}\right\|\left\|S x^{*}-x^{*}\right\|, n \geq 1$. Put $\gamma_{n}=\alpha_{n}(\tau-$ $l \gamma), \delta_{n}=2 \beta_{n} M_{1}+2 \alpha_{n}\left\langle\gamma V x^{*}-\mu B x^{*}, x_{n+1}-x^{*}\right\rangle$. It is easy to see that $\lim _{\sup _{n \rightarrow \infty}} \delta_{n} / \gamma_{n} \leq 0$. Hence, by Lemma 6 , the sequence $\left\{x_{n}\right\}$ converges strongly to $x^{*}$.

\section{Numerical Result}

In this section, we consider the following simple example to demonstrate the effectiveness, realization, and convergence of the algorithms in Theorems 8 and 11.

Example 12. Let $H=\mathbb{R}, C=[1 / 4,+\infty)$. Define $T_{1}: x \mapsto \sqrt{x}$, $T_{2}: x \mapsto x+\pi / 4-\arctan x, S: x \mapsto \sin x$. Take $B=I$ with Lipschitz constant $k=1$ and strongly monotone constant $\eta=$ $1, V x=2 x, \forall x \in H$, with Lipschitz coefficient $l=2$. Give the parameters $\alpha_{n}=1 / 2 n ; \beta_{n}=1 / n^{2}$ for every $n \geq 1$; fix $\mu=1$ and $\gamma=1 / 4$. Then by Theorems 8 and 11, respectively, the sequence $\left\{x_{n}\right\}$ is generated by

$$
\begin{gathered}
y_{n}=\frac{1}{n^{2}} \sin \left(x_{n}\right)+\left(1-\frac{1}{n^{2}}\right) x_{n}, \\
x_{n+1}=P_{C}\left(\frac{1}{4 n} x_{n}+\left(1-\frac{1}{2 n}\right) T y_{n}\right), \\
y_{n}=\frac{1}{n^{2}} \sin \left(x_{n}\right)+\left(1-\frac{1}{n^{2}}\right) x_{n}, \\
x_{n+1}=P_{C}\left(\frac{1}{4 n} x_{n}+\left(1-\frac{1}{2 n}\right) T_{[n]} y_{n}\right) .
\end{gathered}
$$

As $n \rightarrow \infty$, we have $\left\{x_{n}\right\} \rightarrow x^{*}=1$. 
TABLE 1: $x_{1}=2$.

\begin{tabular}{lcc}
\hline$n$ (iterative number) & $x_{n}$ (iterative point) & Errors $(n)$ \\
\hline 50 & 0.9901 & $9.9 \times 10^{-3}$ \\
500 & 0.9990 & $9.9852 \times 10^{-4}$ \\
2000 & 0.9999 & $9.9985 \times 10^{-5}$ \\
\hline
\end{tabular}

TABLE 2: $x_{1}=2$.

\begin{tabular}{lcc}
\hline$n$ (iterative number) & $x_{n}$ (iterative point) & Errors $(n)$ \\
\hline 50 & 0.9902 & $9.8 \times 10^{-3}$ \\
500 & 0.9990 & $9.9814 \times 10^{-4}$ \\
2000 & 0.9999 & $9.9981 \times 10^{-5}$ \\
\hline
\end{tabular}

Let $\omega_{i}=1 / 2, i=1,2$; then we have $T x=(1 / 2)(\sqrt{x}+x+$ $\pi / 4-\arctan x), T_{[n]} x=\sqrt{x}$ if $n$ is odd and $T_{[n]} x=x+\pi / 4-$ $\arctan x$ if $n$ is even. Put $z_{n}=(1 / 4 n) x_{n}+(1-1 / 2 n) T y_{n}$; then (61) is equivalent to

$$
x_{n+1}= \begin{cases}z_{n}, & \text { if } z_{n} \in C, \\ \frac{1}{4}, & \text { if } z_{n} \bar{\epsilon} C .\end{cases}
$$

Using the same method to treat (62), we can get similar equation as the above formula.

Now we turn to numerical simulation using the algorithms (17) and (37), respectively. Take the initial guess $x_{1}=$ 2; using software Matlab R2012, we obtain the numerical experiment results in Tables 1 and 2 .

From the computer programming point of view, the algorithms are easier to implement in this paper.

\section{Acknowledgments}

The authors would like to thank the referee for valuable suggestions to improve the manuscript and the Fundamental Research Funds for the Central Universities (Grant 3122013k004).

\section{References}

[1] P. L. Combettes, "A block-iterative surrogate constraint splitting method for quadratic signal recovery," IEEE Transactions on Signal Processing, vol. 51, no. 7, pp. 1771-1782, 2003.

[2] H. Iiduka and I. Yamada, "A use of conjugate gradient direction for the convex optimization problem over the fixed point set of a nonexpansive mapping," SIAM Journal on Optimization, vol. 19, no. 4, pp. 1881-1893, 2008.

[3] H. Iiduka, "Fixed point optimization algorithm and its application to power control in CDMA data networks," Mathematical Programming, vol. 133, no. 1-2, pp. 227-242, 2012.

[4] L. C. Ceng, Q. H. Ansari, N. C. Wong, and J. C. Yao, "Implicit iterative method for hierarchical variational inequalities," Journal of Applied Mathematics, vol. 2012, Article ID 472935, 14 pages, 2012.

[5] L. C. Ceng, Q. H. Ansari, and J. C. Yao, "Some iterative methods for finding fixed points and for solving constrained convex minimization problems," Nonlinear Analysis, vol. 74, no. 16, pp. 5286-5302, 2011.
[6] H. Iiduka, "Iterative algorithm for solving triple-hierarchical constrained optimization problem," Journal of Optimization Theory and Applications, vol. 148, no. 3, pp. 580-592, 2011.

[7] G. Marino and H. K. Xu, "Explicit hierarchical fixed point approach to variational inequalities," Journal of Optimization Theory and Applications, vol. 149, no. 1, pp. 61-78, 2011.

[8] Y. H. Wang and W. Xu, "Strong covergence of a modified iterative algorithm for hierarchical fixed point problems and variational inequalities," Fixed Point Theory and Applications, vol. 2013, article 121, 2013.

[9] H. K. Xu, "Viscosity method for hierarchical fixed point approach to variational inequalities," Taiwanese Journal of Mathematics, vol. 14, no. 2, pp. 463-478, 2010.

[10] Y. Yao, Y. J. Cho, and Y. C. Liou, "Iterative algorithms for hierarchical fixed points problems and variational inequalities," Mathematical and Computer Modelling, vol. 52, no. 9-10, pp. 1697-1705, 2010.

[11] M. Tian, "A general iterative algorithm for nonexpansive mappings in Hilbert spaces," Nonlinear Analysis, vol. 73, no. 3, pp. 689-694, 2010.

[12] I. Yamada, “The hybrid steepest-descent method for variational inequality problems over the intersection of the fixed-point sets of nonexpansive mappings," Studies in Computational Mathematics, vol. 8, pp. 473-504, 2001.

[13] M. Tian and L. Y. Di, "Synchronal algorithm and cyclic algorithm for fixed point problems and variational inequality problems in Hilbert spaces," Fixed Point Theory and Applications, vol. 2011, article 21, 2011.

[14] S. N. He and W. W. Sun, "New hybrid steepest descent algorithms for variational inequalities over the common fixed points set of infinite nonexpansive mappings," WSEAS Transactions on Mathematics, vol. 11, no. 2, pp. 83-92, 2012. 


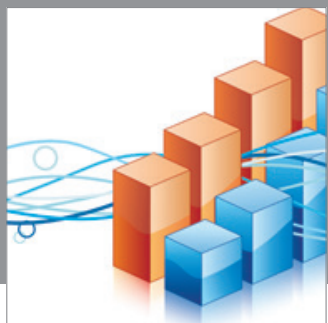

Advances in

Operations Research

mansans

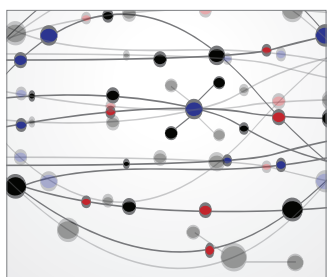

The Scientific World Journal
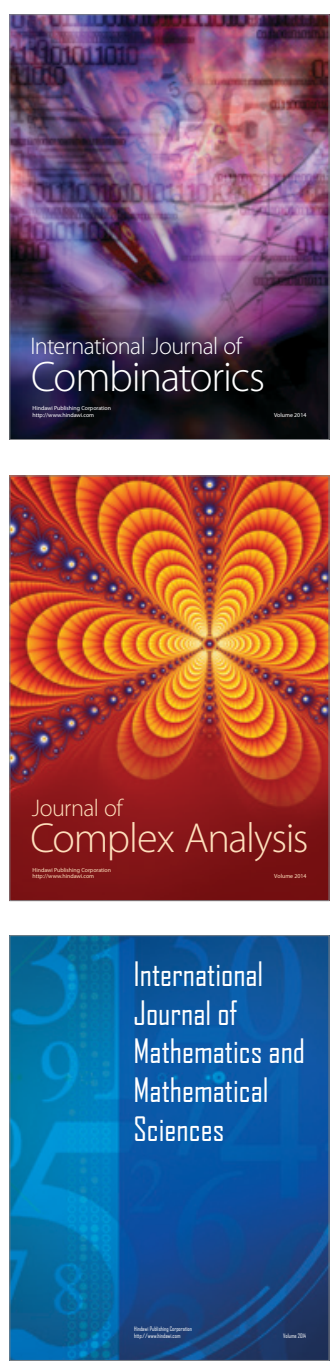
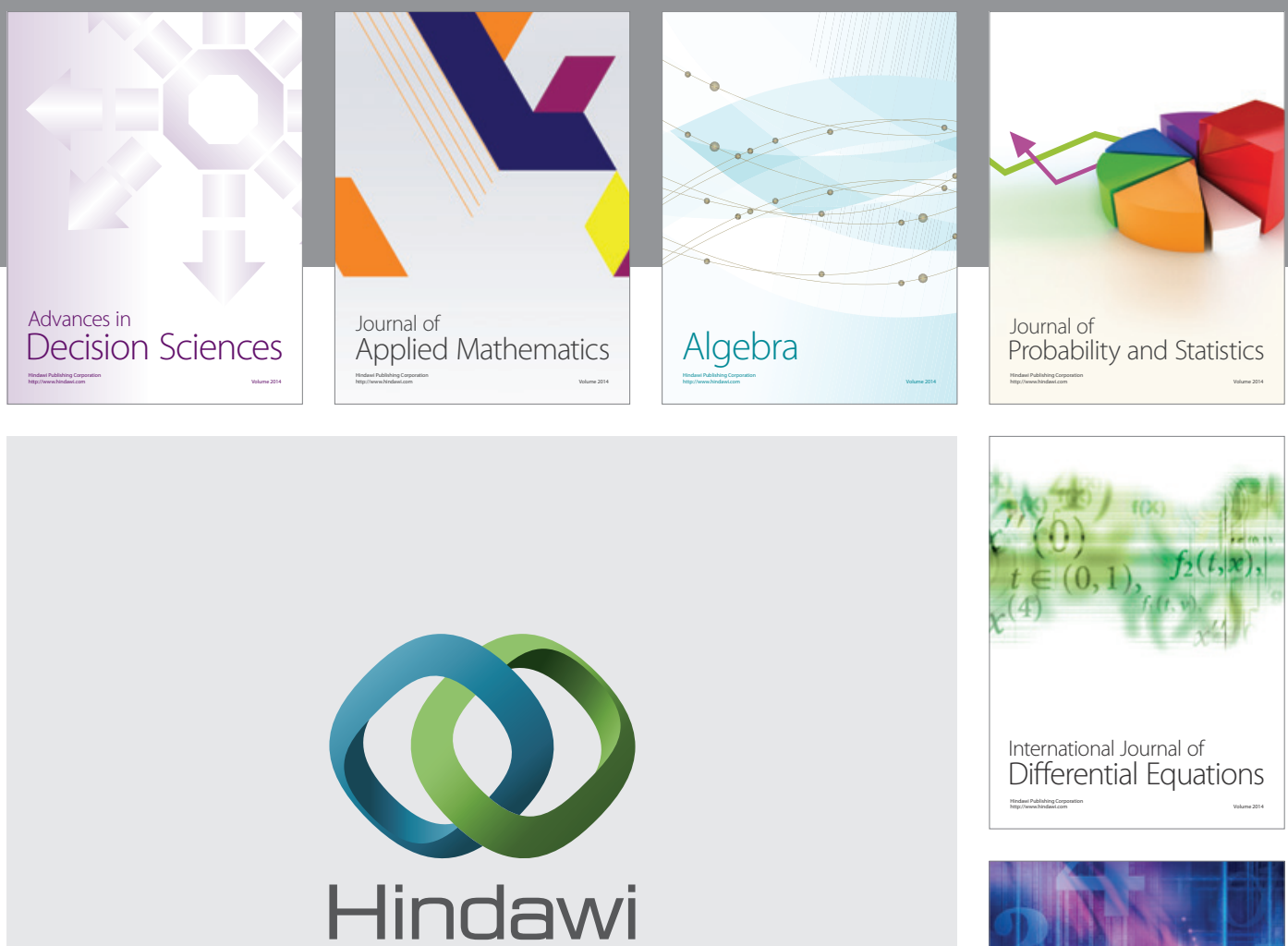

Submit your manuscripts at http://www.hindawi.com
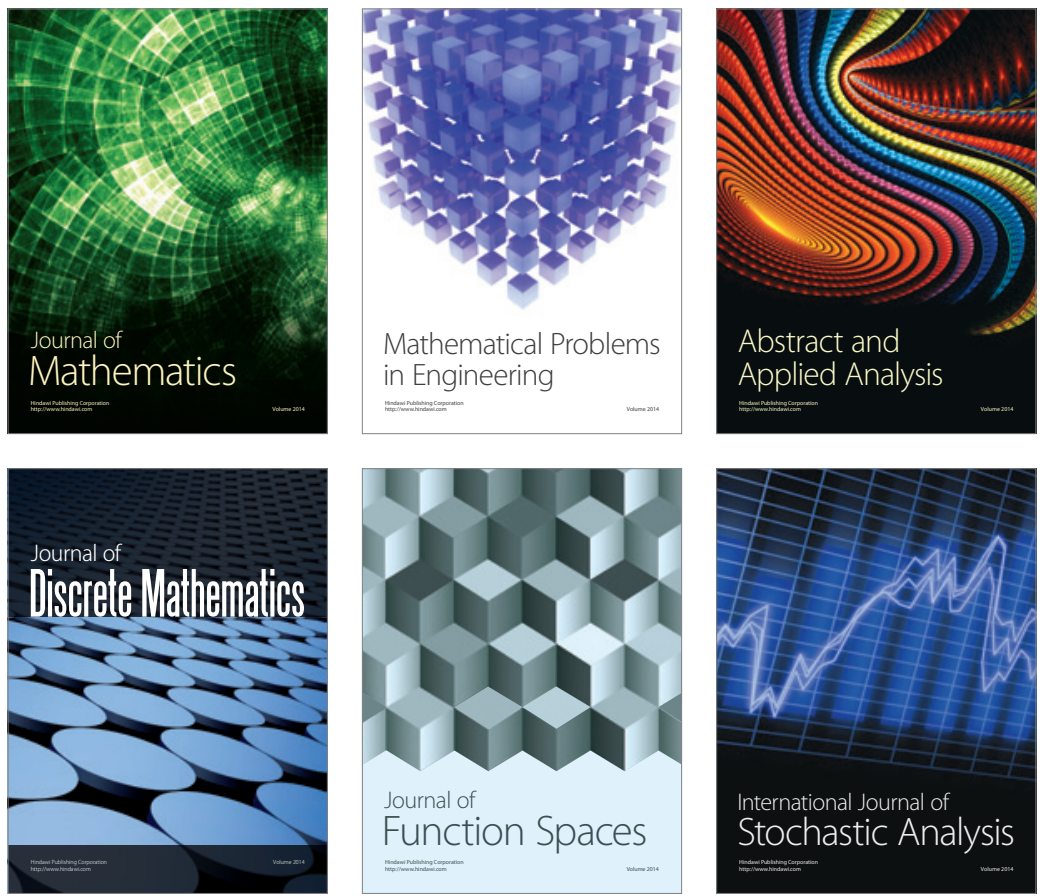

Journal of

Function Spaces

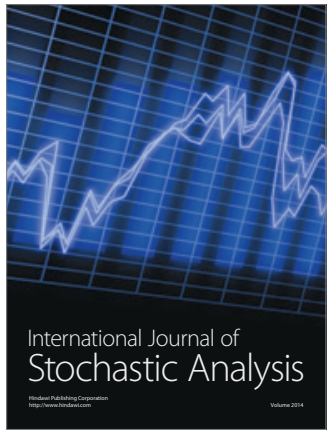

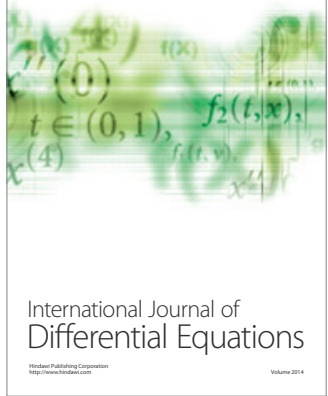
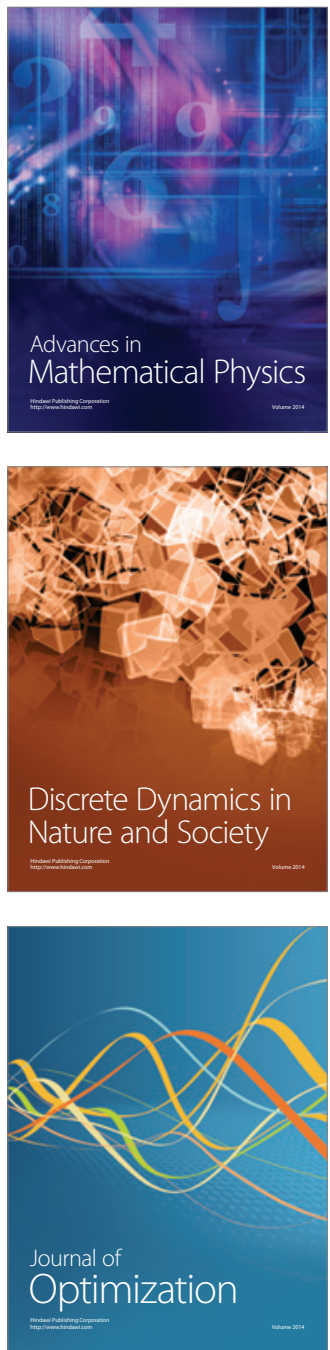